\title{
Pharmacological activities of Triazole analogues as antibacterial, antifungal, antiviral agents
}

\author{
M. Asif \\ Department of Pharmacy, Guru Ram Das (PG) Institute of Management and Technolgy, Dehradun, \\ (Uttarakhand), 248009, India
}

\begin{abstract}
Triazoles and its derivatives possess a great importance in medicinal chemistry and can be used for the synthesis of various compounds with different types of biological activities. The triazole derivatives are possessing divers biological activities such as antibacterial, antifungal, antiviral, antitubercular, anti-inflammatory, analgesic, antitumor, anticonvulsant and some other biological as well as chemical useful compounds. The biological profile of triazole moiety has attracted the attention of many researchers to explore its multiple potential against several biological activities. This article covers the information of some triazoles derivatives having antimicrobial activities. Thus triazole acts as a promising medicinal agent and can be helpful to develop new triazole compounds that could have better efficacy and lesser toxicity.
\end{abstract}

Keyword: Triazole compounds, antibacterial, antifungal, antiviral

\section{INTRODUCTION}

Triazole derivatives possess a large significance in medicinal chemistry and numerous heterocyclic triazole compounds having different biological activities. Triazole is a five membered basic compound, containing two carbon and three nitrogen atoms having molecular formula $\mathrm{C}_{2} \mathrm{H}_{3} \mathrm{~N}_{3}$. It forms a pair of isomeris ${ }^{1-6}$. The 1,2,3-triazole is measured to be the most stable compound in relationship to all other compounds possessing three adjacent nitrogen atoms. Triazole derivatives possess wide variety of pharmacological activities such as antifungal, antibacterial, antiviral, anticancer, anticonvulsant, anti-inflammatory, antioxidant, anti-tubercular, anti-malarial, anti-nociceptive and other anticipated activities ${ }^{7-15}$.<smiles>c1c[nH]nn1</smiles><smiles>c1nc[nH]n1</smiles>

\section{1,2,3-triazole 1,2,4-triazole}

*Corresponding author: aasif321@gmail.com
Triazole is measured to be the most useful constituent and widely used in research purposes as a building block for complex chemical compounds and drug molecules such as antifungal drugs fluconazole, isavuconazole, itraconazole, voriconazole, pramiconazole, and posaconazole. The triazole produced plant protection fungicides like epoxiconazole, triadimenol, propiconazole, metconazole, cyproconazole, tebuconazole, flusilazole paclobutrazol and tazobactam. Various substituted triazole compounds are responsible for producing antibacterial effects and further modifications can be made on it to enhance their antimicrobial effects $^{16-22}$.<smiles>OC(Cn1cncn1)(Cn1cncn1)c1ccc(F)cc1F</smiles>

Fluconazole<smiles>CCC(C)n1ncn(-c2ccc(N3CCN(c4ccc(OC[C@@H]5CO[C@@](Cn6cncn6)(c6ccc(Cl)cc6Cl)O5)cc4)CC3)cc2)c1=O</smiles> 


\section{ANTIMICROBIAL ACTIVITIES}

Bacteria are the simplest and smallest unicellular organism originate independently or in bunches. The huge number of extremely effective and comparatively non-toxic drugs available for the management of bacterial infections has provided tough contest for the medicinal chemist, challenging to synthesis of new antimicrobial agents. These antibacterial agents are classified in two types on the basis of their mode of action as bactericidal and bacteriostatic activities ${ }^{23-30}$.

Antimicrobial and antifungal activity:

The 1-(1H-1,2,4-triazolyl)-2-(2,4-diflurophenyl)3-(4-substituted-1-piperazinyl)-2-propanol derivatives on the basis of the active site of lanosterol $14 \alpha$-demethylase exhibited in vitro antifungal activities. Some of these compounds had higher antifungal activity than fluconazole and some compounds showed good MIC values less than $0.125 \mu \mathrm{g} / \mathrm{mL}$ and more potent than fluconazole. Compound $\mathbf{1}$ and $\mathbf{2}$ had excellent effectiveness against a broad range of pathogenic fungal stains including Aspergillus fumigates ${ }^{31}$. Some ciprofloxacin analogues (3) showed antimicrobial activity. In these compounds ciprofloxacin have been incorporated to the series of Schiff bases of 1,2,4-triazole via Mannich reaction. The compounds showed in vitro antimicrobial activity against gram positive and gram negative bacteria like $B$. subtilis, $K$. pneumoniae, and $P$. aeruginosa at $10 \mu \mathrm{g} / \mathrm{mL}$ concentration. All the compounds showed comparable or superior activity than the reference drug ciprofloxacin ${ }^{32}$. Unsubstituted and 3-substituted-7-aryl-5 $H$-6,7-dihydroimidazo $[2,1-c][1,2,4]$ triazoles (4) were exhibited their antifungal activity against $A$. niger and Fusarium oxysporum. Among these compounds, 7-(3chlorophenyl)-6,7-dihydro-5Himidazo[2,1-c] $[1,2,4]$ triazole-3-thiol was showed the most significant activity ${ }^{33}$. Compounds (5 and 6) were evaluated as antimicrobial agents against against Esherichia coli, P. auroginosa, Yersinia pseudotuberculosis, Klepsiella pneumonia, Enterococcus fecalis, Staphylococcus aureus and Bacillus cereus and ampicillin was used as control antibiotics. These compounds were showed good antibacterial activity against
S. aureus ${ }^{34}$. The 1,2,4-triazole-3-thiol metronidazole derivatives (7a-c and 8a-c) were showed anti microbial agents against G-positive, G-negative bacteria and fungal. With the exception of Clostridium sporogenes the antimicrobial activity was significantly lower than that of the reference antimicrobials $^{35}$. A series of Schiff bases of triazoles showed antibacterial as well as antifungal activity like 5-phenyl,4-(substituted)amino,3mercapto-1,2,4-triazoles (9) showed antimicrobial activity $^{36}$. Schiff's bases N-[1-arylmethelene]1-[8-(triafluromethyl) quinolin-4-yl]-5-methyl1H-1,2,3-triazole-4-carbohydrazide and 1-aryl-4-\{1-[8-(triafluro methyl)quinolin-4-yl]-5methyl-1H-1,2,3-triazole-4-yl \} prop-2-en-1one containing triazole and quinoline moiety (10) were showed their antimicrobial activities against $E$. coli, $S$. aureus, $P$. aeruginosa, $B$. subtilis, Klebsiella pneumonia, Aspergilus flavus, A. fumigates, Candida albicans, Penicillium marneffei, Trichophyton mentagrophytes ${ }^{37}$.

A series of N,N-bis(1,2,4-triazole-1-yl methyl) amine $(\mathbf{1 1}, \mathbf{1 2}, 13)$, condensation of 1(hydroxyl-methyl) with different amines were evaluated for their antifungal activity against budding yeast Saccharomycces cerevisiae and their antibacterial activity was found most active ${ }^{38}$. Antimicrobial activity of some compounds (14, 15, 16), 1-(5-phenylamino- $[1,3,4]$ thiadiazole2-yl)methyl-5-oxo-[1,2,4]triazole and 1-(4phenyl-5-thioxo-[1,2,4]triazol-3-yl)methyl5 -oxo-[1,2,4] triazole derivatives were found to be potent against various microbes ${ }^{39}$. Several 3,6-Disubstituted-1,2,4-triazolo [3,4-b]-1,3,4Thiadiazole and their dihydro analogues (17a-e) were exhibited their antibacterial, antifungal, anti-inflammatory and analgesic activities. The antimicrobial results showed that some of the compounds are active against both G-positive and G-negative bacteria. These compounds also showed good inhibition of growth of the yeast-like $C$. albicans and fungi $A$. niger ${ }^{40}$. Some 4-Aryl triazole derivative (17) were found as antibacterial agents against $B$. cereus, $P$. aeriginosa, K. pneumoniae, Micrococcus flavus and Citrobactor freundii and antifungal agents against Candida tropicalis, C. albicans, Cryptococcus neofomans, Trichospor onbeigelii, and A. flavus ${ }^{41}$. The 3(substituted phenoxymethy)-6-phenyl/substituted phenoxymethyl-1,2,4-triazolo(3,4-B)-thiadiazole 
derivatives (18 and 19) were showed their in-vitro equipotent antibacterial and antifungal activity at MIC of $1 \mu \mathrm{g} / \mathrm{mL}$ when compared with standard drug respectively. These compounds were showed comparable antitubercular activity against M. tuberculosis at MIC of $0.50 \mu \mathrm{g} / \mathrm{mL}$, when compared with standard drug Rifampin and Isoniazid at MIC of $0.25 \mu \mathrm{g} / \mathrm{mL}^{42}$. A series of N-(6-bromo-2-methyl-4-oxoquinazolin-3 (4H)-yl)-2-(4-(3-chloro-2-(substituted phenyl)-4-oxoazetidin-1-yl)-5-(pyridin-4-yl)5-thio)acetamido-1,2,4-triazoles (20a-f) were showed their antibacterial activity against $S$. aureus, E.coli, P. vulgaris, K. pneumonia ${ }^{43}$.<smiles>OC(CN1CCN(c2[R]cccc2)CC1)(Cn1cncn1)c1c(F)cccc1F</smiles>

1<smiles>[R]N1CCN(CC(O)(Cn2cncn2)c2c(F)cccc2F)CC1</smiles>

2<smiles>O=C(O)C1CC(C2CC2)c2cc(N3CCN(Cn4nc(-c5ccccc5)n(/C=C/Br)c4=S)CC3)c(F)cc2C1=O</smiles>

3<smiles>Sc1nnc2n1CCN2c1cccc(Cl)c1</smiles>

4<smiles>COc1ccc(/C=N\n2c(-c3ccccc3)nnc2-c2ccc(C)cc2)cc1</smiles><smiles>COc1cc(OC)c(/C=N/n2c(-c3ccccc3)nnc2-c2ccc(C)cc2)c(OC)c1</smiles>

6<smiles>[R]n1c([Al])nnc1SCCN1C(C)=NCC1N(C)O</smiles><smiles>[R]n1c([Al])nn(CCn2c([N+](=O)[O-])cnc2C)c1=S</smiles>
$\operatorname{Ar}=N^{\prime}$,<smiles>COc1ccc(C)cc1</smiles>

$8 a-e$<smiles>[Al]/C=N\n1c([Al])nnc1S</smiles><smiles>Cc1c(C(=O)N/N=C/c2ccsc2)nnn1-c1ccnc2c(C(F)(F)F)cccc12</smiles> 
<smiles>Brc1cnc(N(Cn2cncn2)Cn2cncn2)c(Br)c1</smiles>

10<smiles>CC(C(=O)O)N(Cn1cncn1)Cn1cncn1</smiles>

11<smiles>c1cc(CN(Cn2cncn2)Cn2cncn2)ccn1</smiles>

12<smiles>[R]c1nn(Cc2nnc(Nc3ccccc3)s2)c(=O)n1N</smiles>

13

14

$15 a-c$

$\mathbf{R}: \mathrm{a}=\mathrm{CH}_{3}, \mathrm{~b}=\mathrm{CH}_{2} \mathrm{C}_{6} \mathrm{H}_{5}, \mathrm{c}=\mathrm{C}_{6} \mathrm{H}_{5}$<smiles>[R]c1nnc2sc([NH2+][CH2])nn12</smiles>

$\mathrm{R}=$ 2-Chloromethoxy phenyl, 3,4Dimethoxy benzyl, 2-Methyl-3-Furanyl; Ar'=2-phenyl-4-quinolinyl, 2-methyl-4quinolinyl, 4-Quinolinyl, 6-Dihydroxy-4-<smiles>[R]c1ccc(-c2nnc(S)n2[R1])cc1</smiles>

$18 \mathrm{R}=-\mathrm{OC}_{2} \mathrm{H}_{5}$<smiles>[R]c1nn2c(COc3ccccc3[R])nnc2s1</smiles>

19 piperydinyl, 5-methoxy-3-indolyl methyl<smiles>Nn1c(S)nnc1-c1ccncc1</smiles><smiles>[R]c1cccc(/C=N\n2c(S)nnc2-c2ccncc2)c1</smiles>

20b<smiles></smiles>

20c<smiles>Cc1nc2ccc(Br)cc2c(=O)n1NC(=O)CSc1nnc(-c2ccncc2)n1/N=C/c1ccccc1</smiles>

20d<smiles>[R]c1ccc(C2SCC(=O)N2C)cc1</smiles>

20 e<smiles>Cc1nc2ccc(Br)cc2c(=O)n1NC(=O)CSc1nnc(-c2ccncc2)[nH]1</smiles>

$20 f$

Figure 1. Structure of some triazole compounds with antimicrobial activities 
The 1,2,4 -triazole derivatives have good antibacterial activity, S-alkylated-1,2,4-triazoles (21) incorporating diphenyl sulphone moieties possess potential antibacterial activity. The pyridyl and naphthyl substituted 1,2,4-triazole (22) was showed antibacterial activity ${ }^{44}$. Some 1,2,4-triazole derivatives $(\mathbf{2 3}, \mathbf{2 4})$ had shown inhibitory activity against some bacteria ${ }^{45,46}$.

Some 1,2,4-triazole derivatives (25) were showed antibacterial activity ${ }^{47}$. The 2,4dihydro-1,2,4-triazol-3-one derivatives (26) were also showed antibacterial activity ${ }^{48}$. The 4-(5-<smiles>[R2]Sc1nnc(-c2cccc(S(=O)(=O)C3C=CC(Br)=CC3)c2)n1[R]</smiles>

$21 \mathrm{R}_{1}-\mathrm{C}_{6} \mathrm{H}_{4}-\mathrm{CH}_{3}(\mathrm{~m}),-\mathrm{C}_{6} \mathrm{H}_{4}-\mathrm{CH}_{3}(\mathrm{p})$; $\mathrm{R}_{2}-\mathrm{C}_{2} \mathrm{H}_{5},-\mathrm{C} 6 \mathrm{H} 4 \mathrm{COCH} 3, \mathrm{CH}_{3} \mathrm{COOCH}_{3}$ $\mathrm{CH}_{3}$<smiles>[R]CNC1C(c2ccccc2)NNC1c1ccncc1</smiles>

$23 \mathrm{R}=-\mathrm{C}_{6} \mathrm{H}_{4} \mathrm{Cl}(\mathrm{p}),-\mathrm{C}_{6} \mathrm{H}_{4} \mathrm{OCH}_{3}(\mathrm{p}),-\mathrm{C}_{6} \mathrm{H}_{2}\left(\mathrm{OCH}_{3}\right)_{3}$<smiles>[R]c1nnc(S)[nH]1</smiles><smiles>[R6]=CCCC=CC</smiles><smiles>CCCC=CCCC</smiles><smiles>CCCCCC(O)CCC</smiles><smiles>[R]C1=CC([R])N(c2ccccc2)C1=C</smiles>

$26 \mathrm{R} 1=\mathrm{H} ; \mathrm{R}_{1}=\mathrm{H} \mathrm{R}_{2}=$ $\mathrm{CH}_{2}$-piperidine, $-\mathrm{CH}_{2}$ piperidine,<smiles>CCN(CC)CC</smiles>

nitro-2-furfurylidene) amino-3-mercapto-5(substituted)-1,2,4-triazoles (27) ${ }^{49}$ and some 1,2,4-triazoles (28) were exhibited antibacterial activity $^{50}$. Antifungal activity of some 1,2,4triazole has been shown, the 4-substituted-5aryl-1,2,4-triazoles (29) ${ }^{51}$ and 1,2,4-triazolium derivatives (30) were exhibited antifungal activity $^{52}$. Some novel mannich bases derived from 3-(4,6-disubstituted-2-thiomethyl-pyrimidyl)4-amino-5-mercapto-1,2,4-triazoles $(\mathbf{3 1})^{53}$ and 1,2,4-triazole derivatives (32) were revealed antifungal activity ${ }^{54}$.<smiles>[R]Sc1nnc([Al])n1-c1cccc2ccccc12</smiles>

22 Ar= 2-Pyridyl, 3-Pyridyl, 4-Pyridyl $\mathrm{R}=-\mathrm{CH}_{2}-\mathrm{C}_{6} \mathrm{H}_{5}$, -<smiles>[R]c1nnc(S)n1N1C(=O)CSC1[Al]</smiles>

$\mathrm{C}_{6} \mathrm{H}_{4} \mathrm{Cl}(\mathrm{p}),-\mathrm{C}_{6} \mathrm{H}_{4} \mathrm{OCH}_{3}(\mathrm{p})$<smiles>[R]/C(=N\n1cnnc1SCCCCCCCC)c1ccc([N+](=O)[O-])o1</smiles>

$27 \mathbf{R}_{\mathbf{1}}=\mathrm{H},-\mathrm{CH}_{3}$<smiles>[Te][Te]Cn1cncn1</smiles>

$28 \mathrm{Ar}=-\mathrm{C}_{6} \mathrm{H}_{5}-\mathrm{C}_{6} \mathrm{H}_{5}(\mathrm{p})$, 2Pyridyl-1,2,4-triazole-4-yl<smiles>C=CCn1c(S)nnc1Br</smiles>

$29 \mathrm{Ar}=4-$ $\mathrm{OH}-\mathrm{C}_{6} \mathrm{H}_{4}-, 2-$ $\mathrm{OH}-5-\mathrm{Cl}-$ $\mathrm{C}_{6} \mathrm{H}_{4}-$<smiles>[Y4]c1ccc(Cn2ncc[n+]2[R])c([X])c1</smiles>

$30 \mathrm{X}_{1}-\mathrm{Cl}, \mathrm{X}=\mathrm{Br}$, $\mathrm{R}=\quad\left(\mathrm{CH}_{2}\right)_{11} \mathrm{CH}_{3}$,

$\left(\mathrm{CH}_{2}\right)_{15} \mathrm{CH}_{3}$ $\mathrm{X}_{1}=\mathrm{F} \mathrm{X}=\mathrm{Br}$, $\mathrm{R}=\left(\mathrm{CH}_{2}\right)_{11} \mathrm{CH}_{3}$<smiles>[R]c1cccc(Nn2c(S)nnc2CSc2nc([R])cc([R])n2)c1</smiles>

$31 \mathrm{R}=-\mathrm{CH}_{3}, \mathrm{R}_{1}=-\mathrm{H}$, $\mathrm{R}_{2}=\quad \mathrm{p}-\mathrm{N}, \mathrm{N}-$ Dimethylphenyl, 3,4-Dimethoxyphenyl,

3,4,5-Trimethoxyphenyl<smiles>[R2]c1ccc(-n2c(COc3ccc(-n4c(-c5ccccc5)nc5ccccc5c4=O)cc3)n[nH]c2=S)c([R])c1</smiles>

$32 \mathrm{R}_{1}=\mathrm{R}_{2}=\mathrm{H} \mathrm{R} \mathrm{R}_{1}=\mathrm{H}, \mathrm{R}_{2}=\mathrm{F} \mathrm{R}$ $=\mathrm{H}, \mathrm{R}_{2}=\mathrm{NO}_{2} \mathrm{R}_{1}=\mathrm{R}_{2}=\mathrm{F}$

Figure 2. Structure of some triazole compounds with antimicrobial activities 
A novel class of cationic anthraquinone analogs 33a-c, compound 33a showed high potency $(\mathrm{MIC}<1 \mathrm{ug} / \mathrm{mL})$ and selectivity against G-positive pathogens including methicillin resistant $S$. aureus (MRSA), while modest activity against G-negative bacteria. Compound 33b and 33c exhibited broad antibacterial activity including MRSA and vancomycinresistant Enterococcus faecalis (VRE), comparable to other available cationic antiseptics ${ }^{55}$. A series of coumarin based 1,2,4 triazol derivatives were exhibited antimicrobial activities in vitro against G-positive bacteria (S. aureus, MRSA, B. substilis and M. luteus), four G-negative bacteria (E. coli, Proteus vulgaris, S. typhi and $S$. dysenteriae) as well as fungi (C. albicans, $S$. cerevisiae and A. fumigatus). Compounds 34 and 35 were displayed stronger antibacterial and antifungal efficacy ${ }^{56}$.

A series of sulphanilamide derived 1,2,3 triazol compounds (36) were displayed in vitro antibacterial and antifungal activities. Compounds 4-amino- $N$-((1-dodecyl-1 $H$-1,2,3-trizol-4-yl) methyl)benzenesulfonamide 36a, 4 amino-N((1-(2,4-dichlorobenzyl)-1H-1,2,3 triazol-4-yl) methyl)-4-aminobenzenesulfonamide $\mathbf{3 6} \mathbf{b}$ and 4-amino- $N$-((1-(2,4-diflurobenzyl)-1H-1,2,3triazol-4-yl) methyl) benzenesulfonamide 36c were found to be most potent compounds against fungal strains ${ }^{57}$. A series of $N, N$ bis $(1,2,4$ triazol1-yl methyl) amines were exhibited antifungal activity against the budding yeast $S$. cerevisiae and antibacterial activity against $E$. coli. Compounds 2,6-bis (bis( 1H-1,2,4-triazol-1-yl) methyl)amino)hexanoic acid 37 and $N, N$ (bis ((1H-1,2,4-triazol-1-yl) methyl)-2 methyl propane-amine 38 were showed strong antifungal and antibacterial activity ${ }^{13}$. Glucosidation of some 4-amino- and 4-arylideneamino-5-(pyridin3-yl)-2,4-dihydro-[1,2,4]-triazole-3-thiones with 2,3,4,6-tetra- $O$-acetyl- $\alpha$-d-glucopyranosyl bromide 39, 40 that gave the corresponding $N$ and $S$ - $\beta$-dglucosides ${ }^{58}$ were showed antimicrobial activity against $A$. fumigatus, Penicillium italicum, Syncephalastrum racemosum, $C$. albicans, $S$. aureus, $P$. aeruginosa, B. subtilis, and E. coli. Some 1-(5-phenylamino-[1,3,4] thiadiazol-2-yl)methyl-5-oxo-[1,2,4]triazoles 41 and 1-(4-phenyl-5-thioxo-[1,2,4]triazol-3-yl) methyl-5-oxo- [1,2,4]triazolederivatives $\mathbf{4 2}$ were displayed antimicrobial properties. Most compounds have antimicrobial activity against one or more microorganism, but no antifungal activity has been observed against yeast like fungi. The inhibitory effect on mycelial growth by three compounds had been observed ${ }^{50}$.

Some 1,3,4-thiadiazol-2-ylmethyl-1,2,4triazoles 43-45 were showed microbial activities. Some compounds of them showed good activity against a variety of microorganisms ${ }^{60}$. A series of 1-[1,2,4-triazol-3-yl] and 1-[1,3,4-thiadiazo 1-2-yl]-3-methylthio-6,7-dihydrobenzo [c] thiophen-4(5H)ones 46 were showed in vitro antimicrobial activity. Some of these compounds exhibited a good activity against $S$. aureus, $S$. epidermidis and Bacillus subtilis ${ }^{61}$. A series of 4-alkyl/aryl-2,4-dihydro-5-((6-(4-bromophenyl) imidazo[2,1-b]thiazol-3-yl)methyl)-3H-1,2,4triazole-3-thiones 47 were showed antibacterial and antifungal activities. These compounds were also showed antitubercular activity against $M$. tuberculosis $\mathrm{H} 37 \mathrm{Rv}^{63}$. Formation of $N$ - and $O$-propargylated quinazoline derivatives from quinazol-4-ones 48 and a series of perfluoroalkyl1H, 1,2,3-triazol-4-yl substituted quinazolines were showed antimicrobial activity and identified as potential compounds ${ }^{63}$.

Substituted coumarin 1,2,4 triazol and sulphanilamide derived 1,2,3 triazol compounds displayed stronger antibacterial action. A series 5-(4-amino substituted-8-(trifluoromethyl) quinolin-3-yl)-4-(un)substituted phenyl-4H1,2,4-triazole-3-thiols (49) from derivatives of 4-hydroxy-8-(trifluoromethyl) quinoline-3carbohydrazide were showed antimicrobial activity against $E$. coli, $S$. aureus, $P$. aeruginosa, $K$. pneumonia and activity was compared with ciprofloxacin as standard drug. The compounds 49a, 49b and 49c showed good activity against all the bacterial strains ${ }^{64}$. A series of sulfanilamidederived 1,2,3-triazole compounds $(\mathbf{5 0}, \mathbf{5 1})$ were showed in vitro antibacterial and antifungal activities against $S$. aureus, E. typhosa, $P$. aeruginosa, S. dysenteriae, B. subtilis, E. coli, as well as $C$. albicans and $C$. mycoderma. The activity of the compounds was compared with the reference drug chloramphenicol. The compounds 50, 51a and 51b bearing dodecyl, 
2,4-dichlorobenzyl and 2,4-difurobenzyl group showed the most potent antibacterial activities against all tested bacterial strains with the MIC values ranging from 32 to $128 \mu \mathrm{g} / \mathrm{mL}^{65}$.<smiles>[R3]N1N=[N+](C)C2C(=O)c3ccccc3C(=O)C21</smiles>

a $\mathrm{R}=-\mathrm{CH}_{2}\left(\mathrm{CH}_{2}\right)_{6} \mathrm{CH}_{3}$;

b $\mathrm{R}=-\mathrm{CH}_{2}\left(\mathrm{CH}_{2}\right)_{10} \mathrm{CH}_{3}$;

c $\mathrm{R}=-\mathrm{CH}_{2}\left(\mathrm{CH}_{2}\right){ }_{14} \mathrm{CH}_{3}$<smiles>[R]n1cc(CNS(=O)(=O)c2ccc(N)cc2)nn1</smiles>

36 (36a) - $\left(\mathrm{CH}_{2}\right)_{11} \mathrm{CH}_{3}$;

(36b); $-\mathrm{CH}_{2} ;(\mathbf{3 6 c}) ;-\mathrm{CH}_{2}$<smiles>Cc1cc(=O)oc2cc(OCc3ccc(Cn4cncn4)cc3)ccc12</smiles>

34<smiles>Cc1cc(=O)oc2cc(OCc3ccc(Cn4cncn4)cc3)c(OCc3ccc(Cn4cncn4)cc3)cc12</smiles>

35<smiles>C=NNCN(CCCCC(CO)N(Cn1cncn1)Cn1cncn1)Cn1cncn1</smiles>

38<smiles>Cn1cnn(-c2nnc(Nc3ccccc3)s2)c1=O</smiles>

41<smiles>Cn1cnn(-c2n[nH]c(=S)n2-c2ccccc2)c1=O</smiles>

39

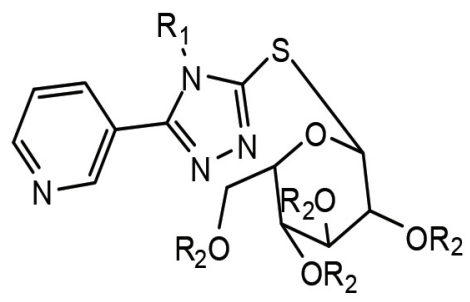

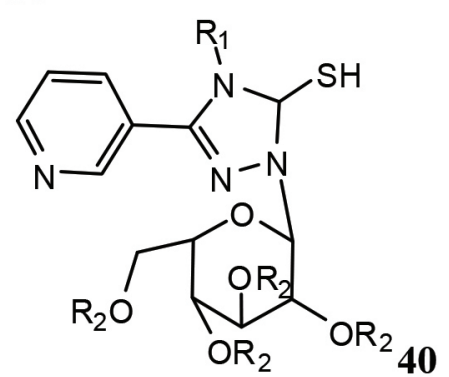

$\mathrm{R}_{1}=\mathrm{H}_{,} \mathrm{NH}_{2}, \mathrm{C}_{6} \mathrm{H}_{5} \mathrm{CH}=\mathrm{N}, 4-$

$\mathrm{CH}_{3} \mathrm{C}_{6} \mathrm{H}_{4} \mathrm{CH}=\mathrm{N}, 4-$

$\mathrm{CH}_{3} \mathrm{OC}_{6} \mathrm{H}_{4} \mathrm{CH}=\mathrm{N}$,

$\mathrm{ClC}_{6} \mathrm{H}_{4} \mathrm{CH}=\mathrm{N} ; \mathrm{R}_{2}=\mathrm{COCH}_{3}, \mathrm{H}$
$4-$

42<smiles>[R]NC(=S)NNC(=O)Cn1nc(-c2ccc(C)cc2)n(N)c1=O</smiles>

43<smiles>[R]n1c(S)nnc1Cn1nc(-c2ccc(C)cc2)n(N)c1=O</smiles>

45<smiles>[R]n1c(Cc2csc3nc(-c4ccc(Br)cc4)cn23)n[nH]c1=S</smiles><smiles>[R]Nc1nnc(Cn2nc(-c3ccc(C)cc3)n(N)c2=O)s1</smiles>

$44 \mathrm{R}=4-\mathrm{FC}_{6} \mathrm{H}_{4}, \mathrm{C}_{6} \mathrm{H}_{5}, 4-\mathrm{OCH}_{3} \mathrm{C}_{6} \mathrm{H}_{4}$<smiles>[Y]c1n[nH]c(C2=C3CCCC(=O)C3=C(SC)C2)n1</smiles>

$46 \mathrm{X}=\mathrm{NR}, \mathrm{S} ; \mathrm{Y}=\mathrm{NH}_{2}, \mathrm{NHR}, \mathrm{SH}, \mathrm{SMe}$<smiles>O=c1c2ccccc2nc(Cl)n1Cc1cn(CCCCC(F)(F)F)nn1</smiles> 
$\mathrm{R}=\mathrm{CH}_{3}, \mathrm{C}_{2} \mathrm{H}_{5}, \mathrm{C}_{3} \mathrm{H}_{7}, \mathrm{C}_{4} \mathrm{H}_{9}, \mathrm{CH}_{2}=\mathrm{CHCH}_{2}, \mathrm{C}_{6} \mathrm{H}_{5} \quad \mathbf{4 8}$<smiles>[R]c1c(-c2nnc(S)n2[R])cnc2c(C(F)(F)F)cccc12</smiles>

$49 \mathbf{R}_{\mathbf{1}}=\mathrm{Ph}, \quad-\mathrm{CH}_{2} \mathrm{Ph}, \quad-$ $\mathrm{CH}_{2} \mathrm{CH}_{2} \mathrm{OMe}$; $\quad \mathbf{R}_{\mathbf{2}}=$ substituted amines; 49a: cyclopropyl amine, $\mathbf{4 9 b}$ : cyclohexyl amine, 49c: 3,3 Dimethyl butylamine. 49d: Piperidine-4-propanol, 49e: Morpholine, 49f: 4-Piperidine morpholine<smiles>CCCn1nncc1CNS(=O)(=O)c1ccc(N)cc1</smiles>

50<smiles>[R]c1cc(-n2nncc2CNS(=O)(=O)c2ccc(N)cc2)cc([R])c1[R]</smiles>

51 51a $\mathrm{R}_{1}=\mathrm{Cl}, \mathrm{R}_{2}=$ $\mathrm{H}, \mathrm{R}_{3}=\mathrm{Cl} ; 51 \mathbf{b} \mathrm{R}_{1}=\mathrm{F}, \mathrm{R}_{2}=$ $\mathrm{H}, \mathrm{R}_{3}=\mathrm{F}$

Figure 3. Structure of some triazole compounds with antimicrobial activities

A derivatives of 1-(substituted biaryloxy)2-(2,4-difluoro phenyl)-3-(1H-1,2,4-triazol-1yl) propan-2-ol derivatives (52) were showed antifungal activity against eight human pathogenic fungi in vitro. Most compounds showed activity between 4- and 64-fold higher than voriconazole against $C$. albicans. Activity suggested that introduction of a biaryloxy side chain greatly improved the antifungal activity of triazole ${ }^{66}$. A series of 4-[(3-substituted-1H- pyrazol- 4-yl) methylene amino]-5-substituted-2-[(4-methyl piperzine-1-yl)methyl]-2H-1,2,4-triazole-3(4H)thiones (53) were showed antibacterial and antifungal activity. Some of the compounds were found to exhibit antimicrobial activity ${ }^{67}$. Various 5-(1-methyl-5-nitro-2-imidazolyl)- $4 \mathrm{H}$ 1,2,4-triazoles (54) were showed in vitro antibacterial and antifungal activities. Two compounds exhibited significant effects against $B$. subtilis at MIC ranges of $0.5-1 \mu \mathrm{g} / \mathrm{mL}$ and moderate effects against $S$. aureus $^{68}$. Various 5-(1-methyl-5-nitro-2-imidazolyl)-4H-1,2,4-triazoles (55) were showed in vitro antibacterial and antifungal activities. Two compounds exhibited significant effects against $B$. subtilis at MIC ranges of $0.5-1 \mu \mathrm{g} / \mathrm{mL}$ and moderate effects against $S$. aureus $^{69}$. A series of 3-[4-(substituted phenyl-5-thioxo-4, 5-dihydro- $1 H$-1,2,4 triazole-3-ylmethoxy)-phenyl]-2-phenyl-3Hquinazoline-4-one (56) was showed antifungal activity. The compound 3-\{4-[-nitrophenyl)5-thioxo-4,5-dihydro-1 $H$-[1,2,4]triazole-3-yl-
methoxy]phenyl\}-2-phenyl-3H-quinaolin-4-one was exhibited good activity against $A$. niger ${ }^{70}$. A series of compound (3) 7-(3-(1H-1,2,3-triazole1-yl)propoxy)-4methyl-2Hchromen-2-one (57) was exhibited in-vitro antifungal activity against strain of $C$. albicans. All compounds except compound (57a) showed moderate antifungal activity while compound (57c) 7-(2(1H-1,2,3-triazole-1-yl)-4-(4-nitrostyryl)-2H chromen-2-one showed antifungal activity as comparable to Ketoconazole ${ }^{71}$. Some 3-( $p-$ substituted anilinoethyl)-4-( $p$-substituted phenyl)5-thioxo-1,2,4-triazoles (58) were showed antifungal activity against $C$. albicans and $A$. niger ${ }^{72}$.

The antibacterial activity of C-5-substituted triazole-oxazolidinones (59) showed antibacterial activity against Mycobacterium smegmatis, B. subtilis, and E. faecalis. The 3-(4-acetyl-phenyl)-5-(1H-1,2,3-triazol-1-yl) methyl)-oxazolidin-2-one was showed 4-fold lower MIC value than isoniazid ${ }^{73}$. A series of heterocyclic systems, triazolo[4,3-a]-quinazolin7-ones (60) [1,2,4,5]-tetrazino[4,3-a]-quinazolin8-ones and indolo[2,3-c][1,2,4]-triazino[4,3-a]quinazolin-8-ones were exhibited antibacterial activity against G-negative bacteria, E. coli, $P$. aeruginosa and G-positive bacteria e.g. $S$. pneumoniae, $B$. subtilis, as well as for antifungal activity against fungal stains, $C$. albicans, $A$. fumigatus, A. flavus and A. niger. Some compounds were showed potent antifungal and antibacterial activity $^{74}$. A series of 2,4-dichloro-5-fluorophenyl 
bearing thiazolotriazoles (51) starting from 3(2,4-dichloro-5-fluorophenyl)-4H-1,2,4-triazole3-thiol were exhibited potent antibacterial activity and some compounds showed significant antifungal activity ${ }^{75}$. The triazole substituted triazolo-pyrimidine derivatives (62) were showed antibacterial activity ${ }^{76}$. The 6,7-dihydro-1,3,4triazolo[1,5-a]-1,3,5-triazin-2-Sulfonamides (63) showed herbicidal and plant growth regulating activity ${ }^{77}$. Some 4 -allyl/amino-5-aryl1,2,4-triazoles (64) were showed antibacterial and antifungal effects against E. coli, B. subtilis, S. enteritidis, S. aureus, A. niger and C. albicans ${ }^{78}$. The 3-[4-(4-substituted phenyl-5-thioxo-4, 5dihydro-1H-1,2,4 triazol-3-ylmethoxy)-phenyl]2-phenyl-3H-quinazolin-4-one (65) were showed in vitro antibacterial activity ${ }^{79}$. The 5-(N-substituted carboxamidomethylthio)-3-(3'-pyridyl)1,2,4-triazole derivatives (66) were showed antibacterial activity ${ }^{80}$.

The 5-(3',4'-dihydro-2',2',8'-trimethyl2'H-1'-benzopyran-7-yloxymethyl)-4-phenyl1,3,4-triazole -3(4H)-thiol (67) was showed significant antifungal activity ${ }^{81}$. The 5-(Nsubstituted carboxamidomethylthio)-3-(3'- pyridyl)- 1,2,4-triazole derivatives (68) showed anti-fungal activity against $C$. albicans and $A$. niger at the concentrations of 50 and $100 \mu \mathrm{g} / \mathrm{mL}$ using Fluconazole as the standard ${ }^{82}$. A series of 3,6-disubstituted-1, 2, 4-triazolo-[3, 4-b]1,3,4-thiadiazoles (69) were showed antifungal activity against $C$. albicans and $A$. niger using Ketoconazole as standard ${ }^{83}$. The Candida fungals were impinged by the new triazole derivatives (70), showed both by in vivo and in vitro antifungal activity. The 1,4-di-substituted-1,2,3triazole compounds with long alkyl chains displayed a good antifungal activity. It was more potent than the reference drugs, ketoconazole, amphoterecin B and fluconazole. The enantiomers are still under development as they are believed to have more potent activity than the racemic compounds. The compound (71) showed excellent activity against $C$. albicans ${ }^{84,85}$. A 2-substituted5-[isopropylthiazole] clubbed 1,2,4-triazole were showed potent antifungal agent. The compound (72), 3-(4-Isopropyl thiazol-2-yl) -6-(4-nitrophenyl)-[1,2,4] triazolo [3,4-b][1,3, 4] thiadiazole ${ }^{86}$ and other compounds (73-76) were also exhibited antifungal activity ${ }^{87}$.<smiles>Cn1c([N+](=O)[O-])cnc1-c1nnc[nH]1</smiles><smiles>[R2]c1ccc(-n2c(COc3ccc(-n4c(-c5ccccc5)nc5ccccc5c4=O)cc3)nn([R])c2=O)c([R7])c1</smiles>

$\mathbf{5 5}$

56<smiles>[R2]c1ccc(/C=C\C2=CC(=O)Cc3cc(OCCCn4cnc([As])n4)ccc32)cc1</smiles><smiles>[R]c1ccc(NCCc2n[nH]c(=S)n2-c2ccc([R2])cc2)cc1</smiles>

(58) $\mathrm{R}_{1}=\mathrm{H}, \mathrm{OCH}_{3}, \mathrm{Br}, \mathrm{Cl} / \mathrm{R}_{2}=\mathrm{H}, \mathrm{CH}_{3}, \mathrm{Br}, \mathrm{Cl}$ 


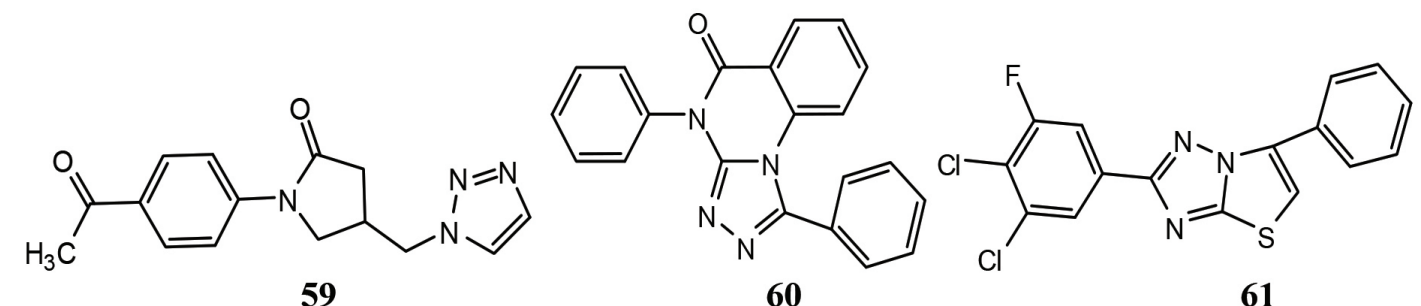<smiles>[R]c1nc2nc(C)nc(C)n2n1</smiles>

62, $\mathrm{R}=$ pyridyl<smiles>[R]c1nc2nc([N+](=O)[O-])nn2c(=O)[nH]1</smiles>

63, $\mathrm{Ar}=($ un $) /$ substituted

60

61<smiles></smiles>

naphthyl, pyridyl; $\mathrm{R}=\mathrm{H}, \quad$ acyl, alkyl,phenyl-alkyl,(un) substituted carbonyl etc. $R_{1}, R_{2}=$ phenyl<smiles>[R]c1ccc(C2C(=S)N([R])N=C2COc2ccc(-n3c(-c4ccccc4)nc4ccccc4c3=O)cc2)c([R])c1</smiles>

65<smiles>[R]N([R])C(=O)CSc1nnc(-c2ccccc2)[nH]1</smiles>

66<smiles>[R]n1c(COc2ccc3c(c2C)OC([R])([R])CC3)n[nH]c1=S</smiles>

67<smiles>[R]N([R])C(=O)CSc1nnc(-c2cccnc2)[nH]1</smiles>

68

$\mathrm{R}=\mathrm{C}_{6} \mathrm{H}_{5}, \mathrm{mCl}-\mathrm{C}_{6} \mathrm{H}_{4}, \mathrm{pCl}-\mathrm{C}_{6} \mathrm{H}_{4}, \mathrm{~m}$-or p- $\mathrm{CH}_{3}-\mathrm{C}_{6} \mathrm{H}_{4}, \mathrm{p}-\mathrm{CH}_{3} \mathrm{O}-\mathrm{C}_{6} \mathrm{H}_{4} \mathbf{R}_{1}=\mathrm{CH}_{3}, \mathrm{C}_{6} \mathrm{H}_{5}, \mathrm{R}_{2}=\mathrm{H}, \mathrm{CH}_{3}$<smiles>CCCCc1cn(CC(O)(Cn2nnc([Al])n2)c2ccc(F)cc2F)nn1</smiles><smiles>c1ccc(C(c2ccccc2)(c2ccccc2)n2cnnn2)cc1</smiles>

71<smiles>[R]O[N+]([O-])c1ccc(N2CN3C(=N2)SCN3c2nc(C(C)C)cs2)cc1</smiles><smiles></smiles><smiles>[R][X]1cccc(C2SCC(=O)C2c2nn3c(-c4ccccc4)nnc3s2)c1</smiles>

75<smiles>[R]c1ccccc1NCC1C(=O)N(c2nn3c(-c4ccccc4)nnc3s2)C1c1ccccc1[R]</smiles>

76

Figure 4. Structure of some triazole compounds with antimicrobial activities 


\section{ANTIVIRAL ACTIVITY}

HIV (retrovirus) resulting in the slow depletion of immune system of the affected human beings resulting in opportunistic infections. Some compounds were evaluated for the antiHIV activity, such as 4-[(1,2-dihydro-2-oxo-3Hindol-3-ylidene)amino]-N(4,6-dimethyl-2pyrimidinyl)-benzenesulphonamide and its derivatives (77) were found active against replication of HIV-1 and HIV-2 in MT-4 cells ${ }^{88}$. Various derivatives of trisubstituted triazoles (78) were act as inhibitors of reverse transcriptase and the two compounds with difference in thio group position were found most active compounds ${ }^{89}$.<smiles>[R]c1ccc2c(c1)/C(=N/c1ccc(S(=O)(=O)Cc3nc(C)cc(C)n3)cc1)C(=O)N2[R]</smiles><smiles>[3H]</smiles><smiles>C=CCC1C(=S)NN=C1COc1ccc(NC(=O)Nc2ccccc2)cc1</smiles>

79
The thiourea derivatives, 5-[(4-amino phenoxy) methyl]-4-alkyl/aryl-2, 4-dihydro-3H-1,2,4 triazole-3-thiones (79) having good activity against cox sacie virus B4, also active against the thymidine kinase positive Varicella zoster Virus $^{90}$. The N-amino-1,2,3-triazole (80) were exhibited antiviral activity against cantalago virus. The ethyl 1-(7-phenyl-2H-3,5,6,7tetrahydro-imidazo[2,1-c][1,2,4]triazol-3-yl) formate $(\mathbf{8 1})$ was effective on human adenovirus 5 (Ad-5) and human enterovirus (Echo-9) replication and the activity against the selected DNA (Ad-5) and RNA (Echo-9) viruses and the cytotoxicity towards normal GMK (Green Monkey Kidney) cells were determined ${ }^{91,92}$.<smiles>Nc1ncnc2ncn(Cc3nn[nH]c3C3CC(O)C(CO)O3)c12</smiles>

78<smiles>CC1C(C(=O)NN)N=NN1Nc1ccc(F)cc1</smiles>

80<smiles>CCOC(=O)C1NN=C2CN(c3ccccc3)CN21</smiles>

Figure 5. Structure of some triazole compounds with antiviral activities

\section{CONCLUSION}

Many different triazole derivatives have been prepared from its useful pharmacological activities. In the present review, reported the different pharmacological activities of triazole derivatives. Triazole derivatives possess a wide range of pharmacological activities such as anticancer, anticonvulsant, antimicrobial, antitubercular, antimalarial, anti-inflammatory, antioxidant, analgesic etc. In general triazole ring, substitution at 1,4 and 1,3 positions with electronegative group will possess more active compounds. Some triazole compounds are in therapeutic uses like, Itraconazole, Voriconazole, Fluconazole, Posaconazole (antifungal), Ribavirin (antiviral), Trapidil (antihypertensive and aasodilator), Rilmazafone (anxiolytic), Nefazodone, Trazodone (antidepressant), Estazolam (sedativehypnotic), Rufinamide (antiepileptic), Anastrozole 
(antineoplastic), Alprazolam (tranquillizer). These diverse pharmacological activities of triazole are the milestone for the new research. Modifications in triazole ring displayed valuable biological activities and these modifications can be utilized to develop more effective agents for future explorations.

\section{REFERENCES}

1. Siddiqui N, Ahsan W, Alam MS, Ali R, Jain S, Azad B, et al. Triazoles: as potential bioactive agents. Inter J Pharm Scie Rev Res. 2011; 8:161-9.

2. Wahi K, Singh A. Triazole: Recent Development and Biological Activities. Asian J Biochem Pharm Res. 2011; 2: 193-205.

3. Unver Y, Meydanal S, Sancak K, Unluer D, Ustabas R, Dugdu E. Synthesis, crystal structure, and antioxidant properties of novel 1,2,4-triazol-5-ones containing 3,4dimethoxyphenyl and 3,4-dihydroxyphenyl moiety. Turk J Chem. 2011;35:265-77.

4. Guantai EM, Ncokazi K, Egan TJ, Gut J, Rosenthal PJ, Smith PJ, et al. Design, synthesis and in vitro antimalarial evaluation of triazole-linked chalcone and dienone hybrid compounds. Bioorg Med Chem. 2010; 18: 8243-56.

5. Mandal SK, Saha D, Jain VK, Jain B. Synthesis and antitubercular activity of some Triazole derivatives of Propyl gallate. Inter J Pharm Sci Res. 2010; 1; 465-73.

6. Devi LN, Devi N, Singh B, Singh MD. Synthesis and Stereochemistry of N4 Amino-1,2,4-Triazoles with unsymmetrical substituents at 3,5-Positions. J Chem Pharm Res. 2011;3:631-8.

7. Xiang L, Xue Q, He-M, Xue X, Zhi H. Synthesis and evaluation of antitumour activities of novel chiral 1,2,4-triazole Schiff bases bearing $\gamma$ - butenolide moiety. Org Med Chem Lett. 2012;2:26.

8. Padmaja A, Rajasekhar C, Muralikrishna A, Padmavathi V. Synthesis and antioxidant activity of disubstituted 1,3,4-oxadiazoles, 1,3,4-thiadiazoles and 1,2,4-triazoles. J Chem Pharm Res. 2012;4(1):294-302.
9. Kemal S, Yasemin U, Dilek U, Esra D, Guulcan K, Fatih C, et al. Synthesis, characterization, and antioxidant activities of new trisubstituted triazoles. Turk J Chem. 2012;36:457-66.

10. Kshirsagar A, Toraskar MP, Kulkarni VM, Dhanashire S, Kadam V. Microwave assisted synthesis of potential antiinfective and anticonvulsant thiosemicarbazones. Inter J Chem Tech Res. 2009;1(3);696-701.

11. Waghamale S, Piste P. Pharmacological activities of triazole, oxadiazole and thiadiazole. Int J Pharm Bio Sci. 2013; 4(3):310-32.

12. Saini MS, Dwivedi J. Synthesis and biological significances of 1,2,4-triazole and its derivatives: a review. Inter J Pharm Sci Res. 2013;4(8):2866-79.

13. Rabea SM, El-Koussi NA, Hassan HY, Fadl TA. Synthesis of 5-phenyl-1-(3-pyridyl)1H-1,2,4-triazole-3-carboxylic acid derivatives potential anti-inflammatory activity. Arch Pharm Chem Life Sci. 2006;339; 32-40.

14. Valentina $P$, Ilango K, Deepthi M, Harusha P, Pavani G, Kumar LS, et al. Antioxidant Activity of Some Substituted-1,2,4-Triazo5-thione Schiff base. J Pharm Sci Res. 2009; 2:74-7.

15. Yan S, Liu Y, Chen Y, Liu L, Lin J, An efficient one-pot synthesis of heterocyclefused 1, 2, 3-triazole derivatives as anticancer agents. Bioorg Med Chem Lett. 2010;20:5225-8.

16. Banday AH, Shameem SA, Gupta BD, Kumar HMS. D-ring substituted 1,2,3-triazolyl 20-keto pregnenanes as potential anticancer agents: Synthesis and biological evaluation. Steroids. 2010;75;801-4.

17. Ibrahim DA. Synthesis and biological evaluation of 3,6- disubstituted $[1,2,4]$ triazolo[3,4-b][1,3,4] thiadiazole derivatives as a novel class of potential anti-tumor agents. Eur J Med Chem. 2009;44:2776-81.

18. Mavrova AT, Wesselinova D, Tsenov YA, Denkova P. Synthesis, cytotoxicity and effects of some 1,2,4-triazole and 1,3,4thiadiazole derivatives on immunocompetent cells. Eur J Med Chem. 2009;44:63-9. 
19. Shehry MF, Abu-Hashem A, El-Telbani EM. Synthesis of 3-((2,4-dichlorophenoxy) methyl)-1,2,4-triazolo(thiadiazoles and thiadiazines) as anti-inflammatory and molluscicidal agents. Eur J Med Chem. 2010; 45:1906-11.

20. Shiradkar M, Suresh Kumar GV, Dasari V, Tatikonda S, Akula KC, Shah R. Clubbed triazoles: A novel approach to antitubercular drugs. Eur J Med Chem. 2006;42(6):80716.

21. Barbuceanu SF, Gabriela LA, Ioana S, Constanatin D, Radu S, Barbuceanu F. New S-alkylated 1,2,4-triazoles incorporating diphenyl sulfone moieties with potential antibacterial activity. J Serb Chem Soc. 2009;74(10):1041-9.

22. Guo LJ, Wei CX, Jia JH, Zhao LM, Quan ZS. Design and synthesis of 5-alkoxy-[1,2,4] triazolo[4,3-a]quinoline derivatives with anticonvulsant activity. Eur J Med Chem. 2009;44:954-8.

23. Zhang J, Redman N, Litke AP, Zeng J, Zhan J, Chan KY, Chang CT. Synthesis and antibacterial activity study of a novel class of cationic anthraquinone analogs. Bioorg Med Chem. 2011;19:498-503.

24. Siddiqui N, Ahsan W, Alam MS, Ali R, Jain S, Azad B, et al. Triazoles: as potential bioactive agents. Inter J Pharm Sci Rev \& Res. 2011; 8(1): 161-169.

25. Amir M, Kumar H, Javed SA, Khan SA, 1,3,4-Oxadiazole/ thiadiazole and 1,2,4triazole derivatives of biphenyl- 4-yloxy acetic acid: Synthesis and preliminary evaluation of biological properties. Eur J Med Chem. 2008;43:2688-98.

26. Pachuta-Stec A, Rzymowska J, Mazur L, Mendyk E, Pitucha M, Rzaczyńska Z. Synthesis, structure elucidation and anti tumour activity of $\mathrm{N}$-substituted amides of 3-(3-ethylthio-1,2,4-triazol- 5-yl) propenoic acid. Eur J Med Chem. 2009;44;3788-93.

27. El-Hawash SA, Habib NS, Kassem MA. Synthesis of some new quinoxalines for evaluation of in vitro antitumor and antimicrobial activities. Arch Pharm. 2006; 339:564-71.
28. Mohamed BG, Abdel-Alim AA, Hussein MA. Synthesis of 1-acyl-2-alkylthio-1,2,4triazolobenz imidazoles with antifungal, anti-inflammatory and analgesic effects. Acta Pharm. 2006;56:31-48.

29. Amir M, Kumar H, Javed SA, Khan SA. 1,3,4-Oxadiazole/thiadiazole and 1,2,4triazole derivatives of biphenyl-4-yloxy acetic acid: Synthesis and preliminary evaluation of biological properties. Eur J Med Chem. 2008;43:2688-98.

30. Karakurt A, Aytemir MD, Stables JP, Ozalp M, Kaynak BF, Ozbey S, et al. Synthesis of Some Oxime Ether Derivatives of 1-(2Naphthyl)-2-(1,2,4-triazol-1-yl)ethanone and their anticonvulsant and antimicrobial activities. Arch Pharm. 2006;339:513-20.

31. Chun QS, Wan NZ, Hai TJ, Yun LS, Min Z, Jun Z, et al. Design, Synthesis and antifungal activity of novel triazole derivatives. Chem Lett. 2004;15(4):404-7.

32. Jubie S, Sikdar P, Kalirajan R, Gowramma B, Gomathy S, Sankar S, et al. Synthesis and antimicrobial activity of some novel ciprofloxacin analogues. J Pharm Res. 2010; 3:511-3.

33. Sztanke K, Tuzimski T, Rzymowska J, Pasternak K, Kandefer-Szerszeń M. Synthesis determination of the lipophilicity, anticancer and antimicrobial properties of some fused 1,2,4-triazole derivatives. Eur J Med Chem. 2018;43:404-19.

34. Mevlut S, Nurhan G, Sengiil A K, Neslihan D. Synthesis of some novel 3,5-diaryl-1,2,4triazole derivatives and investigation of their antimicrobial activities. Turk J Chem. 2007;31;315-26.

35. Haythem A, Ibrahim M, Amal G, Mohammad S. Synthesis and antimicrobial activity of new 1,2,4-triazole-3-thiol metronidazole derivatives. Monatsh Chem. 2010; 141: 471-8.

36. Rao G, Rajasekran S, Attimarad M. Synthesis and anti-microbial activity of some 5phenyl-4- substituted amino-3-mercapto (4H), 1,2,4-triazoles. Ind J Pharm Sci. 2000; 47:475-7. 
37. Holla B, Mahalinga M, Karthikeyan M, Poojary B, Akberali P, Kumari N. Synthesis, characterization andantimicrobial activity of some substituted 1,2,3-triazoles Eur J Med Chem. 2005;40:1173-8.

38. Hanane A, Bouchra Q, Abdelkarim A, Rachid T, Nour-eddin B, Abdellah H, et al. Synthesis and biological activity of new triazole compounds. Lett Drug Des Discov. 2010;7:41-5.

39. Demirbas N, Karaoglu S, Demirbas A, Sancak K. Synthesis and Antimicrobial activities of some new 1-(5-phenylamino$[1,3,4]$ thiadiazole-2yl)methyl-5-oxo-[1,2,4] triazole and1-(4-phenyl-5-thioxo-[1,2,4] triazole-3-yl)methyl-5-oxo-[1,2,4] triazole Derivatives. Eur J Med Chem. 2004;39: 793-804.

40. Mathew V, Keshavayya J, Vaidya V, Giles D. Studies on synthesis and pharmacological activities of 3,6-disubstituted-1,2,4-triazolo [3,4-b]-1,3,4-thiadiazoles and their dihydro analogues. Eur J Med Chem. 2007;42: 823-40.

41. Prajapati S, Goswami K, Patal A. Synthesis and characterisation of 4-Aryl thiazole ring system and its antimicrobial activity. Int $\mathrm{J}$ Pharm Bio Sci. 2013;4(1): 803-8.

42. Hunashal R, Satyanarayana D. One pot synthesis of 3-(substituted phenoxy methy) -6-phenyl/substituted phenoxymethyl1,2,4-triazolo(3,4-B)-thiadiazole derivatives as antimicrobial agents. Int J Pharm Bio Sci. 2012;3(4):183-92.

43. Singh I, Kaur H, Kumar S, Kumar A. Synthesis and antimicrobial activity of some new Pyridinyl /Quinazolinyl /Azetidinyl/ Thiazolidinonyl Triazoles. Inter J Pharm Bio Sci. 2010;6(1):1-17.

44. Zamani K, Faghihi K, Tofighi T, Shariatzadeh MR. Synthesis and antimicrobial activity of some pyridyl and naphthyl substituted 1,2,4triazole and 1,3,4-thidiazole derivatives. Turk J Chem. 2004; 28: 95-100.

45. Nurhan G, Mevlut S, Elif C, Ali S, Neslihan D. Synthesis and antimicrobial activities of some new 1,2,4-triazole derivatives, Turk J Chem. 2007;31:335-48.
46. El-Sayed R. Synthesis, antibacterial and surface activity of 1,2,4-triazole derivatives, Grasas Y Aceites. 2006;57(2):180-8.

47. Mudasir RB, Abdul R. Substituted 1,2,4triazoles and thiazolidinones from fatty acids: Spectral characterization and antimicrobial activity. Indian J Chem. 2009;48:97-102.

48. Stefanska J, Struga M, Tyski S, Kossakowski J, Dobasz M. Antimicrobial activity of 2,4dihydro-1,2,4-triazol-3-one derivatives Polish J Microbiol. 2008;57(2):179-82.

49. Holla BS, Kalluraya B, Sridhar KR. Synthesis of 4-(5-nitro-2-furfurylidene) amino-3-mercapto-5-(substituted)-1,2,4triazoles as possible antibacterial agents. Curr Sci. 1987;56(5);236-9.

50. Vesna D, Nada PJ. QSAR study by $1,2,4-$ triazoles using several physicochemical descriptors. Macedonian J Chem and Chem Eng. 2009;28(1):79-89.

51. Katica CR, Vesna D, Vlada K, Dora GM and Aleksandra B. Synthesis, antibacterial and antifungal activity of 4-substituted-5-aryl1,2,4-triazoles. Molecules. 2001;6:815-24.

52. Luo Y, Lu YH, Gan LL, Zhou CH, Wu J, Geng RX, et al. Synthesis, antibacterial and antifungal activities of novel 1,2,4triazolium derivatives. Arch Pharm Chem Life Sci. 2009;342; 386-93.

53. Lingappa B, Girisha KS, Balakrishna K, Rai NS, Kumari NS. Regioselective reaction: Synthesis of novel mannich bases derived from 3-(4,6-disubstituted-2thiomethyl pyrimidyl) -4-amino-5-mercapto1,2,4-triazoles and their antimicrobial properties. Indian J Chem. 2008;47B: 1858-64.

54. Havaldar FH, Patil AR. Synthesis of 1,2,4triazole derivatives and their biological activity. E-J Chem. 2008;5(2):347-54.

55. Shi Y, Zhau C. Synthesis and evaluation of a class of new Coumarin triazole derivatives as potential antimicrobial agents. Bioorg Med Chem Lett. 2011;21:956-60.

56. Wang X, Wan K, Zhou C. Synthesis of novel sulfanilamidederived 1,2,3-triazoles and their evaluation for antibacterial and antifungal activities. Eur J Med Chem. 2010;45:4631-9. 
57. Bay HA, Quaddouri B, Guaadaoui A, Touzani R, Benchat N, Hamal A, et al. Synthesis and Biological Activity of New Triazole Compounds. Drug Design Discov. 2010;7:41-5.

58. Sam KN. Efficient synthesis, structure, and antimicrobial activity of some novel $N$ - and $S$ - $\beta$-dglucosides of 5-pyridin-3-yl-1,2,4triazoles. Carbohydr Res. 2006;341: 2187 99.

59. Demirbas N, Karaoglu SA, Demirbas A, Sancak K. Synthesis and antimicrobial activities of some new 1-(5-phenylamino$[1,3,4]$ thiadiazol-2-yl)methyl-5-oxo-[1,2,4] triazole and 1-(4-phenyl-5-thioxo-[1,2,4] triazol-3-yl)methyl-5-oxo-[1,2,4]triazole derivatives. Eur J Med Chem. 2004;39: 793-804.

60. Demirbas A, Sahin D, Demirbas N, Karaoglu SA. Synthesis of some new 1,3,4-thiadiazol2-ylmethyl-1,2,4-triazole derivatives and investigation of their antimicrobial activities. Eur J Med Chem. 2009;44:2896-903.

61. Tehranchian S, Akbarzadeh T, Fazeli MR, Jamalifar H, Shafiee A, Synthesis and antibacterial activity of 1-[1,2,4-triazol3-yl] and 1-[1,3,4-thiadiazol-2-yl]-3methylthio-6,7-dihydrobenzo $[c]$ thiophen4(5H)ones. Bioorg Med Chem Lett. 2005; 15:1023-5.

62. Guzeldemirci NU, Kucukbasmacı O. Synthesis and antimicrobial activity evaluation of new 1,2,4-triazoles and 1,3,4-thiadiazoles bearing imidazo[2,1-b]thiazole moiety. Eur J Med Chem. 2010;4:63-8.

63. Chandrika PM, Yakaiah T, Gayatri G, Kumar KP, Narsaiah B, Murthy USN, et al. Click chemistry: Studies on the synthesis of novel fluorous tagged triazol4-yl substituted quinazoline derivatives and their biological evaluation-Theoretical and experimental validation. Eur $\mathbf{J}$ Med Chem. 2010;45:78-84.

64. Eswaran S, Adhikari AV, Shetty NS. Synthesis and antimicrobial activities of novel quinoline derivatives carrying 1,2,4triazole moiety. Eur J Med Chem. 2009; 44:4637-47.
65. Wang XL, Wan K, Zhou CH. Synthesis of novel sulfanilamide-derived 1,2,3-triazoles and their evaluation for antibacterial and antifungal activities. Eur J Med Chem. 2010;45:4631-9.

66. Liu P, Zhu S, Xie W. Synthesis and SAR studies of biaryloxysubstituted triazoles as antifungal agents. Bioorg Med Chem Lett. 2008;18:3261-5.

67. Isloor AM, Kalluraya B, Shetty P. Regioselective reaction: synthesis, characterization and pharmacological studies of some new Mannich bases derived from 1,2,4-triazoles. Eur J Med Chem. 2009;44;3784-7.

68. Shafiee A, Sayadi A, Roozbahani MH, Foroumadi A, Kamal F. Synthesis and in vitro antimicrobial evaluation of 5-(1methyl-5-nitro-2-imidazolyl)-4H-1,2,4triazoles. Arch Pharm. 2002;335:495-9.

69. Shafiee A, Sayadi A, Roozbahani MH, Foroumadi A, Kamal F. Synthesis and in vitro Antimicrobial Evaluation of 5-(1Methyl-5-nitro-2- imidazolyl)-4H-1,2,4triazoles. Arch Pharm. 2002;335:495-9.

70. Havaldar FH, Patil AR. Syntheses of 1,2,4Triazole Derivatives and their Biological Activity. Eur J Med Chem. 2008;5:347-54.

71. Kokil RG. Synthesis and In Vitro Evaluation of Novel 1,2,4-Triazole Derivatives as Antifungal Agents. Lett Drug Des Discov. 2010;7:46-9.

72. Siddiqui AA, Amit A. Synthesis of some 1,2,4-triazoles as potential antifungal agents. Indian J Chem. 2005;44 B:838.

73. Demaray JA, Thuener JE, Dawson MN, Sucheck SJ. Synthesis of triazole-oxazolidinones via a one-pot reaction and evaluation of their antimicrobial activity. Bioorg Med Chem Lett. 2008;18:4868-48.

74. Pandey SK, Singh A, Nizamuddin A. Antimicrobial studies of some novel quinazolinones fused with[1,2,4]-triazole, $[1,2,4]$-triazine and[1,2,4,5]- tetrazine rings. Eur J Med Chem. 2009;44:1188-97.

75. Karthikeyan MS. Synthesis, analgesic, antiinflammatory and antimicrobial studies of 2,4-dichloro-5-fluorophenyl containing thiazolotriazoles. Eur J Med Chem. 2009; 44:827-33. 
76. Colanceska RK, Vesna D, Vlado K, Gabor MD, Aleksandra B. Synthesis, antibacterial and antifungal activity of 4-Substituted-5aryl-1,2,4-triazoles. Molecules. 2001;6: 815-24.

77. Freddy HH, Abhay RP. Synthesis of 1,2,4triazole Derivatives and their Biological Activity. E-J Chem. 2008;5(2):347-54.

78. Mali RK, Somani RR, Toraskar MP, Mali KK, Naik PP, Shirodkar PY, et al. Synthesis of some antifungal and anti-tubercular 1,2,4-triazole analogues. Inter J ChemTech Res. 2009;1(2):168-73.

79. Amir M, Javed SA, Kumar H. Synthesis of some newer analogues of 4-hydroxyphenyl acetic acid as potent anti-inflammatory agents. J Chinese Chem Soc. 2008;55: 201-8.

80. Birsen T, Esra K, Erdem Y, Mevlut E. Preparation of 5-aryl-3-alkylthio-1, 2, 4triazoles and corresponding sulfones with anti-inflammatory-analgesic activity. Bioorg Med Chem. 2007;15:1808-14.

81. Ilango K, Valentina P. Synthesis and biological activities of novel 1, 2, 4-triazolo[3, 4-b]-1, 3, 4-thiadiazoles. Der Pharma Chemica. 2010;2(2):16-22.

82. Aher NG, Pore VS, Mishra NN, Kumar A, Shukla PK, Sharma A, et al. Synthesis and antifungal activity of 1,2,3-triazole containing fluconazole analogues. Bioorg Med Chem Lett. 2009;19:759-63

83. Chai X, Zhang J, Hu H, Yu S, Sun Q, Dan Z, et al. Design, Synthesis and biological evaluation of novel triazole derivatives as inhibitors of cytochrome P450 14 ademethylase. Eur J Med Chem. 2009; 24:1913-20.

84. Mitscher LA. Bacterial topoisomerase inhibitors-quinolone and pyridone antibacterial agents. Chem Rev. 2005;105(2): 559-92.

85. Todoulou OG, Papadaki-Valiraki AE, Ikeda S, Clercq E. Synthesis and antiviral activity of some new $1 \mathrm{H}-1,2,4$-triazole derivatives. Eur J Med Chem. 1994;29: 611-20.

86. Wuest F, Tang X, Torsten K, Pietzsch J, Mavanur S. Synthesis and cyclooxygenase inhibition of various (aryl-1,2,3-triazole1-yl) methanesulfonylphenyl derivatives. Bioorg Med Chem. 2009;17(3):1146-51.

87. Rezaei Z, Khabnadideh S, Pakshir K, Hossaini Z, Amiri F, Assadpour E. synthesis, and antifungal activity of triazole and benzotriazole derivatives. Eur J Med Chem. 2009;44(7):3064-7

88. Selvam P, Chandramohan M, De Clercq E, Witvrouw M, Pannecouque C. Synthesis and anti-HIV activity of 4-[(1,2-dihydro-2-oxo3H-indol-3-ylidene)amino]-N-(4,6-dimethyl2- pyrimidinyl) benzene sulphonamide and its derivatives. Eur J Pharm Sci. 2001;14: 313-6.

89. Hluhanich R, Kutty N, Liclican AC, McColl DJ, Squires NH, Lansdon ER. Triazole derivatives as non-nucleoside inhibitors of HIV-1 reverse transcriptase-Structureactivity relationships and crystallographic analysis. Bioorg Med Chem Lett. 2008; 18:1131-4.

90. Peterson LB, Blagg BSJ. Click chemistry to probe Hsp90: Synthesis and evaluation of a series of triazolecontaining novobiocin analogues. Bioorg Med Chem Lett. 2010; 20:3957-60.

91. Cheng ZY, Li WJ, He F, Zhou JM, Zhu $\mathrm{XF}$. Synthesis and biological evaluation of 4-aryl-5- cyano-2H-1,2,3-triazoles as inhibitor of HER 2 tyrosine kinase. Bioorg Med Chem. 2007;15:1533-8.

92. Saini MS, Dwivedi J. A Review: Synthesis and Biological significances of 1,2,4-triazole and its derivatives. Int J Pharm Sci Res. 2013:4(8);2866-79.

93. Mali RK, Somani RR, Toraskar MP, Mali KK, Naik PP, Shirodkar PY, et al. Synthesis of some Antifungal and Anti-tubercular 1,2,4-Triazole Analogues. Inter J ChemTech Res. 2009;1(2):168-73. 